\title{
A ECONOMia Circular Gera OPORTUNidAdes para AS CADEIAS PRodutivas IMPACTADAS PELA PANDEMIA? UMA PERSPECTIVA BRASILEIRA
}

Istefani Carísio de Paula (istefanicpaula@gmail.com) - Universidade Federal do Rio Grande do Sul. Ghissia Hauser (ghissia@ hotmail.com) - Universidade Federal do Rio Grande do Sul.

Aline Cafruni Gularte (alinecgularte@ gmail.com) - Universidade Federal do Rio Grande do Sul. Isabella Rodrigues da Cunha e Paula (isabela.veloso@ @otmail.com)

João Pedro Maciel Jaeger (joaomjaeger@gmail.com) - Universidade Federal do Rio Grande do Sul.

\section{RESUMO}

A Economia Circular (EC) é uma alternativa ao modelo de produção linear vigente, mas em que medida pode ser a resposta para a crise imposta às cadeias produtivas afetadas pela pandemia Covid-19? O objetivo deste trabalho foi promover um evento composto por palestras com especialistas brasileiros a respeito das oportunidades que a EC pode gerar para as cadeias produtivas impactadas pela pandemia. $\mathrm{O}$ caminho metodológico escolhido foi a consulta a quatro especialistas por meio de uma live, sob uma adaptação do método Delphi. A live foi gravada, transcrita e seu conteúdo analisado. Em relação à questão formulada, os resultados indicam que, a geração de emprego, a inovação e diversificação de produtos, serviços e processos típicos da EC, são oportunidades que fazem frente ao desemprego vigente. A abordagem do tema por meio de live demonstrou ser um instrumento útil em pesquisas exploratórias de temas emergentes. Outras oportunidades de investigação em EC emergiram da análise e são apresentadas ao final do artigo.

Palavras-chave: Economia Circular, live, Covid-19, cadeias produtivas, Delphi 


\section{INTRODUÇÃO}

De acordo com relatório da Confederação Nacional da Indústria (CNI, 2018) pensar a longo prazo é essencial para o enfrentamento de crises. A Economia Circular (EC) é uma alternativa ao modelo de produção linear vigente trazendo benefícios para a sociedade e o meio ambiente, regenerando sistemas naturais, eliminando resíduos e poluição desde o princípio e mantendo produtos e materiais em uso pelo maior tempo possível (EMF, 2020).

Havana (2021) argumenta que a EC seria especialmente relevante em momentos de crise econômica global, como a atual pandemia de Covid-19, que surgiu na China, em dezembro de 2019 (SATOMI et al., 2020). A falta de conhecimento científico e o elevado grau de disseminação da doença contribuíram para que governantes e gestores seguissem as recomendações da Organização Mundial da Saúde (OMS). Uma das recomendações foi o isolamento social para diminuir a contaminação da população. Essa medida impactou diretamente as cadeias produtivas e as empresas "não essenciais" tiveram que suspender suas atividades (BERNARDES; SILVA; LIMA, 2020).

Dentre as consequências, destacam-se demissões, redução de salários, queda da renda dos trabalhadores informais e uma modificação do consumo. Esta crise é classificada como um dos maiores desafios de política econômica já enfrentados e, no Brasil, soma-se às dificuldades decorrentes da ausência de um plano claro de enfrentamento à crise (SEBRAE, 2020).

Os pesquisadores do Núcleo de Inteligência para Projetos e Sistemas - CNPq (NIProS - dgp.cnpq.br/dgp/espelhogrupo/664428) têm buscado responder perguntas tais como: em que medida a EC pode ser a resposta para a crise imposta às cadeias produtivas afetadas pela pandemia? Um caminho metodológico para explorar um dado tema, especialmente quando se trata de um tema inovador, é a consulta formal a experts. Assim, o objetivo deste trabalho foi promover um evento composto por palestras com especialistas a respeito das oportunidades que a EC pode gerar para as cadeias produtivas impactadas pela pandemia.

Recentemente tem crescido a realização de transmissões ao vivo (live) feitas por meio de redes sociais. Entre suas vantagens está o grande alcance geográfico e a facilidade de organização, podendo atingir um número quase ilimitado de espectadores. O espaço formal escolhido para as palestras dos especialistas foi a estruturação de uma live e a análise de conteúdo gerou o resultado obtido nesta investigação. 
As contribuições percebidas neste trabalho incluem: (i) testar novos recursos como meio de exploração de temas inovadores em pesquisa; (ii) trazer evidências a respeito de oportunidades em EC que minimizem os impactos da pandemia no Brasil; (iii) gerar conteúdo para fins de pesquisa; e, (iv) sensibilizar e ampliar o conhecimento para os espectadores.

\section{REVISÃO TEÓRICA}

Durante a pandemia Covid-19, a restrição de movimento de milhões de pessoas, vem impactando diretamente as cadeias de abastecimento e expondo o sistema produtivo a variações que provocam recessão econômica. Além disso, a crise atual evidenciou as deficiências do sistema linear que causam degradação ambiental, mudanças climáticas, poluição e perda de biodiversidade (EMF, 2020).

Políticos e investidores europeus se unem para definir iniciativas visando determinar a direção da recuperação econômica, a curto e longo prazos, além de reconfigurar os papéis do Estado e dos atores do mercado para os próximos anos (EMF, 2020). Uma das estratégias de recuperação econômica é a EC que tem como objetivo a eliminação de resíduos através do design superior de materiais, produtos, sistemas e modelos de empresas, proporcionado novas formas de crescimento, redefinindo economias em crise, atenuando riscos de crises futuras (EMF, 2020), integrando as atividades econômicas e o bem-estar ambiental à luz da sustentabilidade (MURRAY; SKENE; HAYNES, 2017).

No Brasil, uma pesquisa revelou que para $70 \%$ dos gestores de empresas industriais, o tema EC ainda é novidade. No entanto, ao assinalarem sobre práticas relacionadas ao EC, 76,5\% das empresas responderam que já empregam alguma dessas práticas (CNI, 2018).

Para tratar de temas emergentes como a EC, diferentes abordagens investigativas podem ser adotadas, tais como: levantamento de literatura, entrevistas com especialistas, entre outros. As etapas iniciais de uma investigação em temática emergente, pode depender da opinião de especialistas, sempre que houver escassez de evidências na própria literatura.

Com o isolamento e distanciamento social um novo cenário para as relações sociais vem sendo criado e ocasionando novos desafios para a comunicação. No campo das mídias sociais, o termo "Live streaming" refere-se a mídias que são simultaneamente gravadas e transmitidas em tempo real. Kramer (2016), descreve Live streaming como uma "ferramenta chave de marketing e comunicações que ajuda marcas a alcançarem sua audiência online" a baixo custo. Além disso, a Live streaming pode incluir cobertura de vários eventos de diferentes temáticas, utilizando uma web browser ou aplicativos como Stream Yard. Nesta 
pesquisa, o conceito de Live streaming foi empregado como recurso investigativo em pesquisa exploratória com especialistas.

\section{METODOLOGIA}

Dois propósitos nortearam a decisão de empregar como recurso de pesquisa exploratória uma live realizada por meio de redes sociais. O primeiro propósito foi promover depoimento de especialistas visando compreender em que medida a EC pode ser a resposta para a crise imposta às cadeias produtivas afetadas pela pandemia. $\mathrm{O}$ segundo, foi promover a divulgação do tema. Este último não será discutido neste artigo.

\subsection{Método de trabalho}

A criação da live foi embasada na metodologia Delphi que permite reunir opiniões de especialistas separados geograficamente possibilitando fazer leituras da realidade, compreensão de fenômenos e principalmente orientar tomadas de decisões (MARQUES; FREITAS, 2018). Segundo Powell (2003), a primeira etapa do método Delphi é a escolha dos especialistas, com foco em grupos heterogêneos com variedade de informações a serem partilhadas, buscando encontrar um equilíbrio entre a imparcialidade, interesse nos assuntos, experiência, áreas de especialidades e perspectivas em relação ao assunto ou problema (MARQUES; FREITAS, 2018). A segunda etapa é a análise de dados das palestras, que pode ser feita de forma qualitativa e/ou quantitativa. Neste estudo, optou-se pela realização da análise qualitativa utilizando o método de análise de conteúdo (Bardin, 1977). Optou-se por abordar nesta pesquisa apenas a rodada inicial de questionamentos aos especialistas.

\subsubsection{Busca exploratória e seleção de experts em EC}

Leituras prévias e pesquisa Desk (diferentes mídias, notícias de jornais) permitiram identificar personalidades proeminentes na área de EC no país. Também foram obtidas indicações de nomes por meio de acadêmicos da área.

\subsubsection{Planejamento da live}

A organização da live ocorreu de acordo com as seguintes etapas: (a) definição da temática; (b) elaboração de briefing; (c) elaboração e submissão de convite aos experts; (d) definição de plataformas digitais para transmissão; (e) definição das mídias de divulgação; (f), elaboração de material de divulgação e divulgação, (g) detalhamento do programa da live, (h) 
comunicação com os experts antes da live, (i) inscrições de espectadores, (j) contratação de divulgação por meio de redes sociais e (k) divulgação e inscrição dos interessados.

\subsubsection{Execução e análise de conteúdo da live}

A live contou com a participação de 4 especialistas brasileiros em EC por meio da plataforma Stream Yard® (https://streamyard.com/), transmitida via Youtube ${ }^{\circledR}$ (https:// https://www.youtube.com/). Ao final, os espectadores receberam um certificado de participação.

Como unidade de análise utilizou-se a própria questão temática da live: "em que medida à EC é resposta para a crise imposta às cadeias produtivas afetadas pela pandemia Covid-19?”. Como unidade de contexto considerou-se a própria origem e formação dos especialistas e seus vieses estratégico, tático e operacional em relação à EC. As palestras foram transcritas e a partir delas, definidas categorias organizadas em planilha Excel®. Além disso, mapas mentais foram elaborados para cada especialista (www.miro.com) e, posteriormente, as categorias puderam ser quantificadas com auxílio de tabelas dinâmicas do Excel®. Nesta análise considerou-se categoria quando dois ou mais especialistas abordaram o mesmo tema.

\section{RESULTADOS E DISCUSSÃO}

Os resultados aqui apresentados se concentram essencialmente na etapa de análise de conteúdo (seção 3.1.3). A live foi realizada no dia 30 de março de 2021, às 19h. Estavam inscritos no evento 527 pessoas, dos quais 215 (40,8\%) assistiram à live. Destes, 59,5\% são mulheres e 40,5\% homens. A faixa etária predominante na live foi dos participantes entre $25 \mathrm{e}$ 34 anos, representando 49,6\%. A busca por especialistas brasileiros em EC trouxe à tona 4 profissionais que aceitaram participar do desafio de responder à questão proposta no Briefing:

Em que medida a Economia Circular pode ser resposta para crise imposta às cadeias produtivas afetadas pela pandemia? Considerando que a abordagem da EC opera sob a tríade da sustentabilidade e que conta com os benefícios da logística reversa, podemos perceber em casos reais de que forma os princípios da EC podem minimizar os impactos da pandemia sobre as cadeias produtivas no mercado?

Buscou-se trazer para o evento profissionais com diferentes visões, os especialistas $2 \mathrm{e}$ 4 trazendo uma perspectiva mais estratégica para o tema, enquanto os especialistas 1 e 3 uma perspectiva tática e operacional. O perfil dos especialistas é apresentado no Quadro 1. 
A ordem de apresentação seguiu uma lógica crescente, trazendo a perspectiva operacional e tática, primeiramente, e em seguida a perspectiva estratégica. Cada especialista teve um tempo de dez minutos para realizar sua fala.

QUADRO 1 - Perfil dos especialistas

\begin{tabular}{|c|c|c|c|}
\hline Especialistas & Formação & & Área de atuação \\
\hline $\begin{array}{c}\text { Especialista } \\
1\end{array}$ & $\begin{array}{l}\text { Engenheiro } \\
\text { Ambiental }\end{array}$ & $\begin{array}{l}\text { Doutor em Engenharia } \\
\text { de Produção pela USP. }\end{array}$ & $\begin{array}{l}\text { Fundador de uma startup de EC, } \\
\text { membro da Comissão Especial de } \\
\text { Estudos em EC, Consultor em EC, } \\
\text { pesquisador e professor. }\end{array}$ \\
\hline $\begin{array}{c}\text { Especialista } \\
2\end{array}$ & $\begin{array}{l}\text { Engenheiro } \\
\text { químico }\end{array}$ & $\begin{array}{l}\text { Mestre em Engenharia } \\
\text { Ambiental pela } \\
\text { University of Surrey. }\end{array}$ & $\begin{array}{l}\text { Fundador do Núcleo de EC, membro } \\
\text { da Plataforma de EC das Américas. } \\
\text { Professor. }\end{array}$ \\
\hline $\begin{array}{c}\text { Especialista } \\
3\end{array}$ & Arquiteto & $\begin{array}{l}\text { Mestre em Gestão para } \\
\text { Eco-Inovação pela } \\
\text { Université de Versailles }\end{array}$ & $\begin{array}{l}\text { Atua em 'advisory and development' } \\
\text { com experiência em projetos } \\
\text { internacionais para implementação } \\
\text { de modelos baseados em EC. }\end{array}$ \\
\hline $\begin{array}{c}\text { Especialista } \\
4\end{array}$ & Economista & $\begin{array}{l}\text { Mestre em } \\
\text { Administração Pública } \\
\text { pela EBAPE/FGV. }\end{array}$ & $\begin{array}{l}\text { Representante de uma Organização } \\
\text { Industrial. }\end{array}$ \\
\hline
\end{tabular}

A live iniciou com o Especialista 1 (Apêndice A), de perfil acadêmico, com a temática intitulada "Economia Circular: estratégia de retomada pós-pandemia". Em sua apresentação, expôs os cenários pós e pré-pandemia, explicando porque a EC é necessária nos dois contextos. Também apresentou os riscos que a continuidade da Economia Linear representa para a sociedade, para o meio ambiente e para as empresas, citando problemas como a recessão e o aquecimento global. O palestrante finalizou expondo os benefícios da geração de empregos, temporários ou não, a partir das inovações propostas pela EC, além da redução de impacto ambiental.

Na segunda apresentação do Especialista 2 (Apêndice B), de perfil empreendedor, foi abordado sobre a temática intitulada: "Como a Economia Circular gera oportunidades para as cadeias produtivas". Em sua explanação, apresentou exemplos práticos de situações que são decorrentes da economia linear, expondo a necessidade de mudanças de hábitos, do pensamento sistêmico e do compartilhamento, citando o HUB de EC desenvolvido por eles. Também apresentou casos práticos em que o HUB, como facilitador, proporcionou o compartilhamento de informações e resultou em colaboração entre empresas de diferentes setores.

Em seguida, o Especialista 3 (Apêndice C), apresentou a palestra intitulada "O potencial integrativo da Economia Circular como um (re)orientador da recuperação pós- 
pandemia”. Em sua apresentação expôs a aplicação da ferramenta Roadmap, o impacto da pandemia nas estratégias das empresas e na transição para a EC. Também apresentou a EC como instrumental para mudança em diversas escalas, assim como os fatores que a EC necessita para impactar efetivamente as cadeias.

Finalizando a rodada de palestras, o Especialista 4 (Apêndice D), representante de uma organização industrial, expôs as ações desenvolvidas por essa organização no âmbito da EC no Brasil e apresentou as agendas e estratégias da entidade relacionadas à sustentabilidade.

Ao realizar a análise de conteúdo das quatro palestras, identificou-se nove categorias que foram abordadas por pelo menos dois palestrantes (Figura 1). A relação entre as categorias e o que cada especialista abordou pode ser visto no Apêndice E.

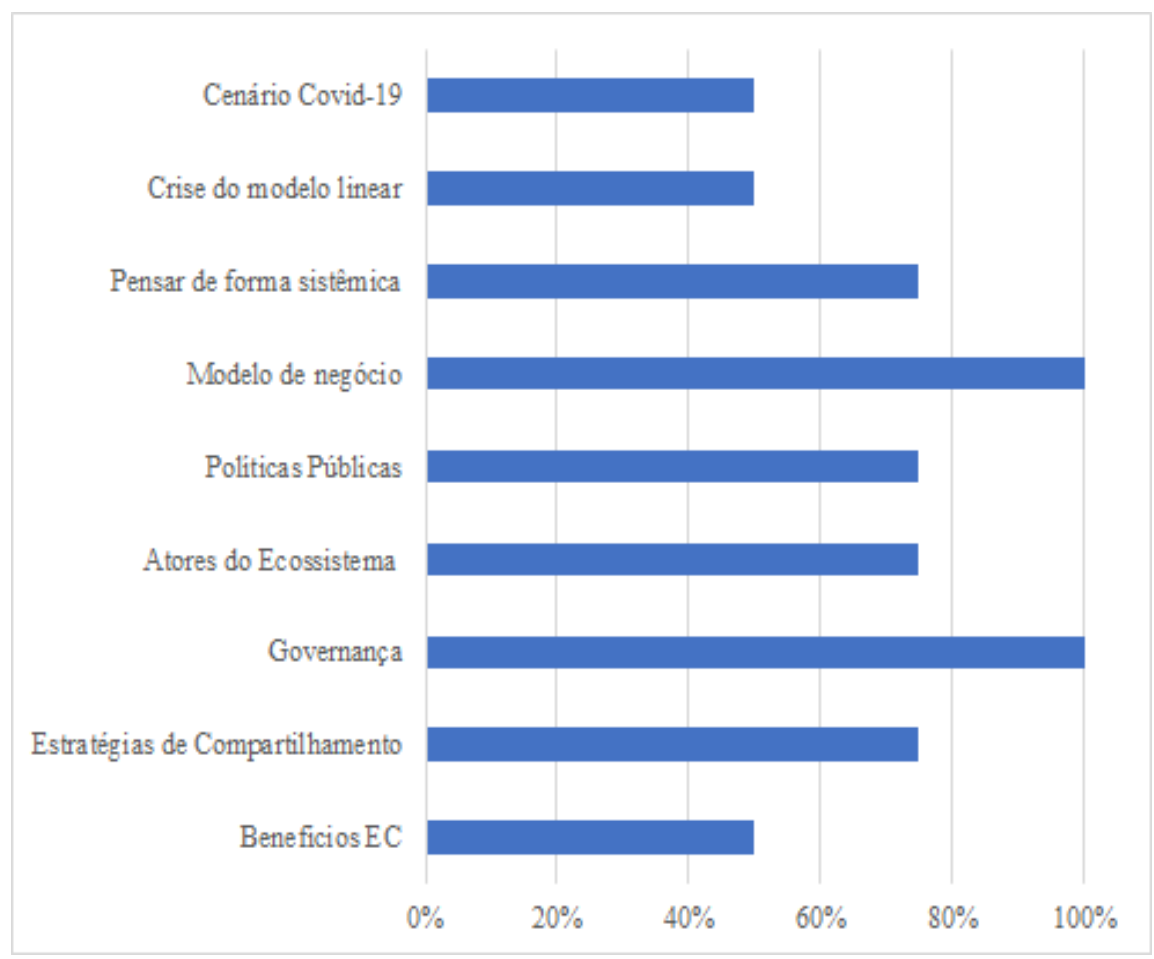

FIGURA 1 - Categorias abordadas entre dois ou mais especialistas

O gráfico (Figura 1) mostra que das nove categorias, duas foram abordadas por todos os especialistas (100\%), quatro categorias foram criadas a partir das falas de 3 especialistas (75\%) e duas categorias, a partir das falas de 2 especialistas (50\%). As duas categorias que se destacaram foram sobre Modelos de Negócio da EC e Governança do sistema.

A crise do modelo linear resultou na construção do conceito de EC (EMF, 2020) que demanda um pensamento sistêmico, com novos modelos de negócio articulando com os atores do ecossistema de forma a atuarem de forma colaborativa e compartilhada com um 
sistema de governança bem estruturado. Além disso, é fundamental a existência de políticas públicas específicas para dar suporte ao ecossistema.

Na opinião dos especialistas, a pandemia (i) Covid-19 é inegável a repercussão global, com impactos sociais, econômicos, políticos e culturais sem precedentes na história recente. $\mathrm{O}$ Especialista 2, ressalta que "[...] a pandemia veio para nos mostrar todas as nossas fragilidades: fragilidade da infraestrutura urbana, dos sistemas de saúde e inclusive do nosso sistema imunológico. Também aponta o papel da EC em meio a pandemia “[...] a EC já estava posta como sendo um novo modelo de desenvolvimento macroeconômico e agora ficou cada vez mais evidente".

Os especialistas abordaram a evidente e preocupante (ii) Crise do Modelo Linear. EMF (2020), ressalta que a crise atual ampliou as deficiências no sistema linear no qual a extração de recursos e produção desordenada de resíduos vem causando degradação ambiental, mudanças no clima, poluição e perda de biodiversidade. O Especialista 1, expõe a urgência da transição do Linear para o circular, apontando que os "[...] modelos de negócios totalmente lineares, ou seja, de extração, produção, uso e descarte, em um mundo com recursos finitos, não fazem mais sentido".

Diante desta crise e a necessidade de transacionar o modelo de negócio atual para um novo cenário de circularidade, é essencial que os gestores comecem a (ii) pensar de forma sistêmica, pois o caminho para a transição não é tão claro e são necessárias pessoas visionárias para que a transição seja rápida e assertiva (JANSSENS, 2021). Pensar de forma sistêmica inclui considerar a interdependência entre suas partes, lembrando que cada um dos componentes se comporta como um sistema individual, cujo resultado é maior do que o resultado que as unidades poderiam ter se funcionassem independentemente.

Também, se faz necessário que o (iii) Modelo de Negócio seja reestruturado, pois a circularidade exige mudanças na forma como as empresas geram valor, entendem e fazem negócios (PIERONI, 2019). Os gestores são compelidos a interagir dentro de um ecossistema de atores, passando de uma lógica operacional centrada na empresa para uma centrada no sistema, requerendo repensar os modelos de negócios existentes, a fim de permitir uma dissociação entre a criação de valor e o consumo de recursos (BOCKEN et al., 2016). O Especialista 2 destaca sobre a importância de entender as conexões inusitadas neste sistema e que às vezes a solução para um negócio circular de um sistema pode estar em outro sistema.

As (iv) Políticas Públicas, entram neste sistema com um papel crucial na transição destes modelos de negócios, elas refletem a importância de ações do governo, através de 
incentivos ao mercado e regulamentações, assegurando condições de estabilidade para a transição para a EC (RIBEIRO et al., 2014). Além disso, o Especialista 4 comenta sobre outro ponto relevante que traz a questão da necessidade das empresas terem um incentivo econômico, estes que estão presentes nas políticas públicas ambientais, mas poucas são as implementações. Como exemplo, países, como a China, incorporaram diretrizes de EC em suas políticas públicas como a base de seu desenvolvimento econômico, aumentando o conceito nas mentes dos formuladores de políticas e ONG's ocidentais (MURRAY et al., 2017).

Os (v) Atores do Ecossistema, são representantes da EC (governo, empresas, mercado, setor produtivo, consumidores, sociedade) responsáveis por realizar ações concretas e no sentido da conscientização geral da importância da EC para o meio ambiente, para as empresas e para a sociedade (JABBOUR et al., 2020). O Especialista 3, fala quem são esses atores e da necessidade deles saberem operar esse novo sistema "[...] eu preciso de profissionais, diretores, governantes, líderes, profissionais de diversos níveis e a comunidade em geral, os comércios, sabendo operar esse novo sistema operacional que vai ser EC". Paula et al. (2011) afirmam que no escopo da relação entre atores como empresas e universidade para $\mathrm{P} \& \mathrm{D}$, a contexto é caracterizado por grandes incertezas, muitas assimetrias de conhecimento e informação entre os parceiros, altos custos de transação na troca de conhecimentos, e restrições para financiamento de atividades de produção e intercâmbio de conhecimento. Estas precisam ser superadas no contexto da EC.

É primordial que um sistema de EC tenha uma (vi) Governança para conduzir os atores dos ecossistemas de EC, visando a satisfação dos objetivos coletivos. Percebe-se a necessidade de práticas de governança que promovam integração e facilitem mudanças no estilo de vida urbano e nos padrões de consumo (FRATINI, 2019). O Especialista 3 traz um exemplo de ferramenta que auxilia na governança de um sistema: “[...] precisamos ter roadmaps que sejam capazes de guiar os setores, os arranjos produtivos, mas também guiar as atividades no nível mais interno." Além disso, Paula et al. (2020) discutem que em contextos colaborativos a governança tem que considerar a indissociabilidade entre "confiança e colaboração".

Os novos produtos resultantes de um sistema circular devem ser analisados estrategicamente. Estudos como a durabilidade, capacidade de reuso e o (vii) Compartilhamento de riscos e investimentos, resultará em benefícios ao sistema. O sistema será beneficiado com uma maior lucratividade dos negócios, proximidade com o cliente, o 
qual passará a dar preferência à qualidade ao invés da quantidade, à formação de sistemas circulares, com menor consumo de recursos e que seja focado no desempenho (CNI, 2018). Posto isto, alguns destes benefícios da EC podem ser percebidos tanto na integração entre atividades econômicas, mas também no bem-estar ambiental de forma sustentável (MURRAY et al., 2017).

\section{CONSIDERAÇÕES FINAIS}

Em relação à resposta à pergunta central da pesquisa, os especialistas indicaram que “sim, a EC é uma estratégia que responde à crise imposta pela pandemia Covid-19". Na opinião dos especialistas com viés estratégico, a pandemia trouxe urgência à uma agenda governamental que já vinha se desenhando no mundo, e consequentemente, também no Brasil. Os especialistas 1 e 2 apontaram como benefícios da EC, geração de emprego, inovação e diversificação de produtos, serviços e processos, como oportunidades que fazem frente ao desemprego trazido pela crise. Não se pode, no entanto, esquecer que o contexto brasileiro ainda é permeado por problemas basilares como infraestrutura, energia, saneamento, legislação, cultura, que podem frear a velocidade de instalação de sistemas produtivos circulares, segundo especialista 4. A definição de políticas públicas viabilizadoras é ao mesmo tempo urgente e essencial.

Os especialistas apontaram o papel das universidades na formação de profissionais qualificados e lideranças para a EC. No que se refere ao aprendizado, percebe-se que lives são trabalhosas, mas tecnologicamente fáceis de serem realizadas. Os próprios integrantes do grupo de pesquisa se capacitaram e a implementaram. A divulgação em redes sociais exigiu recursos financeiros empregados no Facebook $₫$, Instagram ${ }^{\circledR}$ e LinkedIn $®$. Para atingir o maior número possível de espectadores no país, também foram usadas as redes de relacionamento dos próprios integrantes do NIProS e de parceiros. Aspectos como tempo de fala, tipo de mediação necessária, como divulgar melhor não fizeram parte do escopo deste artigo. Como limitação não foi apresentado no escopo do trabalho os resultados da rodada de debate realizada após as palestras e como oportunidades futuras propõem-se a realização de novos eventos tratando de temas como a relação entre a EC e a Indústria 4.0 e os desafios enfrentados na implementação da EC no Brasil. 


\section{AGRADECIMENTOS}

Aos especialistas por compartilharem sua experiência e à equipe do NIProS pelo envolvimento neste desafio.

\section{REFERÊNCIAS}

BARDIN, L.. Análise de conteúdo. Lisboa, Edição 70, 1977.

BERNARDES, J. R.; DE SOUSA SILVA, B. L.; LIMA, T. C. F. Os impactos financeiros da Covid-19 nos negócios. Revista da FAESF, vol.4, 2020.

BOCKEN, N. M. P.; SHORT, S. W.; RANA, P.; EVANS, S. A literature and practice review to develop sustainable business model archetypes. Journal of Cleaner Production. 2014

CNI. Confederação Nacional da Indústria. Economia circular: oportunidades e desafios para a indústria brasileira. Confederação Nacional da Indústria, Brasília, CNI, 2018. Disponível em: <https://edisciplinas.usp.br/pluginfile.php/5532421/mod_resource/content/1/Economia\%20Circular_CNI_2018.p $\underline{\text { df }}>$

EMF. Ellen MacArthur Fundation. The circular economy: a transformative Covid-19 recovery strategy. EMF, 2020. Disponível em: <https://www.ellenmacarthurfoundation.org/publications/covid-19>. Acesso em: 25 mai. 2021.

FRATINI, C. F.; GEORG, S.; JØRGENSEN, M. S. Exploring circular economy imaginaries in European cities: A research agenda for the governance of urban sustainability transitions. Journal of cleaner production, vol.228, p. 974-989, 2019.

HAVANA, L. P. N. Economia circular em face da pandemia, alternativa ou necessidade? Disponível em: <https://www.prensalatina.com.br/index.php?o=rn\&id=38747\&SEO=economia-circular-em-face-da-pandemiaalternativa-ou-necessidade> Acesso em: 26 mai. 2021.

JABBOUR, C. J., et al. Stakeholders, innovative business models for the circular economy and sustainable performance of firms in an emerging economy facing institutional voids. Journal of Environmental Management, vol.266, 2020.

JANSSENS, L.; KUPPENS, T.; VAN SCHOUBROECK, S. Competences of the professional of the future in the circular economy: Evidence from the case of Limburg, Belgium. Journal of Cleaner Production, vol. $281,2021$.

KRAMER, B. How Live-Streaming is Going to Crush it in 201. SocialMediaToday, 2016. Disponível em: <https://www.socialmediatoday.com/social-networks/how-live-streaming-going-crush-it-2016>. Acesso em: 16 jun 2021.

MARQUES, J. B. V.; FREITAS, D. Método DELPHI: caracterização e potencialidades na pesquisa em Educação. Pro-Posições, v. 29, n. 2, p. 389-415, 2018.

MURRAY, A.; SKENE, K.; HAYNES, K. The Circular Economy: An Interdisciplinary Exploration of the Concept and Application in a Global Context. J Bus Ethics, vol.140, pág. 369-380, 2017.

PAULA, I.C., YANG, S.; KORZENOWSKI, A. CORTIMIGLIA, M.N. Stakeholders Analysis Tools to Support the Open Innovation Process Management - Caso Study International Conference on Engineering Design. Technical University of Denmark, 2011. 
PAULA, I.C.; CAMPOS, E.A.R.; PAGANI, R.N.; GUARNIERI, P.; KAVIANI, M.A. Are collaboration and trust sources for innovation in reverse logistics? Insights from a systematic literature review. Supply Chain Management, v. 25, 2, 2020.

PIERONI, M. P. P.; MCALOONE, T. C.; PIGOSSO, D. C. A. Business model innovation for circular economy and sustainability: A review of approaches. Journal of Cleaner Production, 2019.

POWELL, C. The Delphi technique: myths and realities. Journal of Advanced Nursing, vol.41, n.4, pág. 376$382,2003$.

RIBEIRO, F. de M.; KRUGLIANSKAS, Isak. A Economia Circular no contexto europeu: Conceito e potenciais de contribuição na modernização das políticas de resíduos sólidos. XVI Encontro Internacional sobre Gestão Empresarial e Meio Ambiente (ENGEMA). São Paulo, 2014. Disponível em: $\langle$ http://www.engema.org.br/XVIENGEMA/473.pdf>

SATOMI, E. et al. Fair allocation of scarce medical resources during COVID-19 pandemic: ethical considerations. Einstein, São Paulo, vol.18, 2020. Disponível em: < https://www.scielo.br/j/eins/a/vTdGYcZkxvFYjZGZH9cNN4v/?format=pdf\&lang=pt>. Acesso em: 25 mai. 2021.

SEBRAE. Serviço Brasileiro de Apoio às Micro e Pequenas Empresas. Análise da crise e impactos para os pequenos negócios. $\quad 2020 . \quad$ Disponível <https://www.sebrae.com.br/Sebrae/Portal\%20Sebrae/UFs/ES/Sebrae\%20de\%20A\%20a\%20Z/RELAT\%C3\%9 3RIO\%20IMPACTO\%20COVID\%20-\%20CORRETO.pdf>. Acesso em: 24 mai. 2021.

TASHA, R. Why Shia LaBeouf's \#AllMyMovies was so successful. The Verge, 2016. Disponível em: <https://www.theverge.com/2015/11/16/9731856/shia-labeouf-all-my-movies-live-stream>. Acesso em: 16 jun. 2021.

\section{APÊNDICES}

Apêndice A - Mapa Mental Especialista 1. Link

Apêndice B - Mapa Mental Especialista 2. Link

Apêndice C - Mapa Mental Especialista 3. $\underline{\text { Link }}$

Apêndice D - Mapa Mental Especialista 4. Link

Apêndice E - Quadro das categorias levantadas na Análise de Conteúdo. Link 